\title{
Diffusion of Technology for Organizational Effectiveness: An Exploratory Study of the Procurement Department of a Multi-National Energy Company in Trinidad and Tobago
}

\author{
Prahalad Sooknanan' ${ }^{1}$, Jim Leung Chee ${ }^{2}$ \\ ${ }^{1}$ University of Trinidad and Tobago, O'Meara Campus, Arima, Trinidad and Tobago \\ ${ }^{2}$ Telecommunication Services of Trinidad and Tobago (TSTT), Port of Spain, Trinidad and Tobago \\ Email: Prahalad.sooknanan@utt.edu.tt
}

Received 26 August 2014; revised 25 September 2014; accepted 20 October 2014

Academic Editor: Clark Callahan, Brigham Young University, USA

Copyright (C) 2014 by authors and Scientific Research Publishing Inc.

This work is licensed under the Creative Commons Attribution International License (CC BY). http://creativecommons.org/licenses/by/4.0/

(c) O) Open Access

\begin{abstract}
This study investigates the diffusion of technology within a homogenous corporate cultural context according to age and educational level to determine how these variables determine the rate of diffusion. Based on the findings, the conventional communication models seem to apply. Specifically, the study shows that in the particular organizational context, age and education level impact on diffusion of technology. Younger and higher educated individuals seem to have a greater affinity to communication and use new communication technologies in their personal lives as well as professional lives which lend itself to possible early adoption or innovation. The study concludes, in the particular context, while hiring younger and more educated staff can possibly enhance the innovation and adoption process, it is perhaps equally, if not more important, to engage innovators from the wider organization to hasten the diffusion process.
\end{abstract}

\section{Keywords}

Diffusion, Innovation, Organizational Effectiveness, Procurement, Multi-National Energy Company 


\section{Introduction}

New communication technologies are rapidly becoming a necessity for organizational effectiveness in today's global economy. In order to be competitive, companies must utilize new communication technologies to leverage global position and resources to exploit new supply markets that are geographically dispersed and not necessarily close in time and space. Technologies such as computers and interfaces with mobile phone technology facilitate longer working hours while allowing the flexibility to provide for a better quality of life and life/work balance. This flexibility is critical for Procurement Departments to conduct business effectively with supply markets in time zones that are different by four (4) hours or more. Such networked technologies facilitate communication beyond the workplace without being "chained" to the office at unrealistic times. However, energy-based companies operating on the Point Lisas Industrial Estate in Trinidad and Tobago seem quite conservative in adopting technologies such as home/work networked computers, text messaging, conferencing technology and "Blackberry" type technology.

This case study explores the challenges in adopting and implementing technology at the Procurement Department of a leading nitrogen company in Trinidad. Specifically, the study seeks to identify the factors that hinder adoption and implementation with the hope of offering solutions. The Procurement Department arguably requires the greatest use of communication techniques within any organization. These departments are normally the negotiating arms of the organization, requiring it to communicate with external organizations on behalf of other departments. In order to be competitive, the process needs to take place locally as well as across borders of several countries with virtually no limit on the geographic and cultural diversity. This is particularly true of multinational companies operating within the new "global village". In addition, the Procurement Department has as its clients every employee in the company. Efficient and effective internal communication is therefore paramount to success.

Generally, companies need to embrace communication technologies such as e-mail as a primary communication method rather than a periphery method in order to reap the full benefits of the technology. Some may even view e-mail as formal documentation, that is, the equivalent of a formal memo, rather than an informal "regular" communication method. This thinking limits the potential of the technology.

New technologies such as text and instant messaging also need to be embraced quickly to facilitate faster and remote communications. Technologies e.g. "Blackberry" etc. and "network to home" arrangements also need to be made widely available. This study will consider the Technology Adoption model (Davis, 1989; Kelleher, 2006) and Diffusion of Innovation model (Rogers, 1995) and attempt to determine what demographics within the Procurement Department might influence the rate of adoption of such new technologies within the department.

The findings of the study have the potential to benefit energy companies in particular, and organizations in general, by identifying potential barriers to adoption and implementation of such communication technologies. If a company decides to pursue communication technology as a strategic advantage, the study may succeed in identifying barriers that may be encountered and hence assist in offering strategies to mitigate such barriers. In addition, the body of research in diffusion of technology in the developing world, and Trinidad and Tobago in particular, is limited. This research will therefore add to the existing body of literature.

The objective of the study was to identify the demographics that hinder technology acceptance in the Procurement Department of a nitrogen company in Trinidad and Tobago. Specifically, the aim was to isolate a cultural context (national and organizational) in order to study the effects of age and educational level as potential barriers or enablers to adoption and implementation and offer possible solutions or approaches to creating strategic and competitive advantage via communication technology.

To this end, the following research questions (RQs) were posed:

RQ 1: Do age and education demographics influence technology acceptance and adoption within a particular corporate environment?

RQ 2: Can infusing a group with younger more educated staff assist accelerate the diffusion process?

RQ 3: Does usage of the technology at home correlate with more positive attitudes towards such technologies in the work place?

Potentially, the study was expected to reveal that there is no correlation between the demographics tested or establish a correlation between the demographics of age and education level with the tendency to be innovators or early adopters (Rogers, 1995). More particularly, it was expected to show that younger, more educated em- 
ployees would be the innovators or natural early adopters. Further, it was anticipated that technology use at home or in personal life would correlate with positive attitudes towards the use of emerging and new communication technologies in business.

\section{Literature Review}

The classic and generally accepted sender, message, channel and receiver model (or SMCR) (DeFleur et al., 2005) still applies today, but the media or channels are getting more complex, plentiful and posing their own encoding and decoding challenges. In addition, different, new and sometimes more complex aspects of "noise" arise and can inadvertently affect the success of the message transmission (Shannon \& Weaver, 1965; DeFleur, 1993) and even determine whether the medium is ultimately used by the wider population. Some new concepts of barriers (or noise) in using new technologies such as SMS texting or e-mail might be lack of shared meaning or symbolism between the parties or perhaps selectiveness or bias. This was illustrated by DeFleur (1993) in two examples. The first illustrated emoticons being used in e-mail to express emotion. This is a new standard symbol and is not shared by all instinctively. The other was in reference to the interpretation of appropriateness of e-mail for certain types of communication between students and professors.

There is little discussion, however, as to whether the appropriateness has to do with the sender and receiver having a shared understanding of appropriateness and/or symbolism and whether demographics might be affecting this. While DeFleur et al. (2005) proposed rules for different media, one may question whether these norms are truly a result of embracing the new technologies and acceptance of the new symbolism and context or whether these are norms that are being superimposed on the new media by the generation in which the current power resides. Therefore, these "norms" may actually be internal barriers to be overcome or in fact may become irrelevant over time.

While DeFleur et al. (2005) discuss the aspects of "communicating between cultures" and "using media to communicate interpersonally", there was little reference to the impact of differing age groups and further any interconnecting discussion on the effect of age or education on the new communication technologies. Further, there was no in-depth discussion about how new media norms like mobile phones and e-mail may be different in diverse cultures, much the same as non-verbal symbols may be different.

Gladwell (2001) postulates the diffusion of trends and the adoption of ideas and products generally as a theory of "epidemics" reaching the "tipping point" in his illustration of the exponential diffusion of fax machines in the US. This has direct relation to the spread and adoption of technologies both communication and otherwise on a large scale. The "tipping point" of Gladwell's theory of the epidemic is analogous to the spread of new ideas and things and is classified into three rules: the Law of the Few, the Stickiness Factor, and the Power of Context. Gladwell (2001), like Rogers (1995), divided the population into groups. However, the former took the view that the epidemic spread and adoption is dependent on Connectors (those that have large social networks), Mavens (the keepers of information) and Salesmen (those that sell the ideas). He asserted that in order to have rapid spread, your resources should be focused on these three groups. Gladwell (2002: 256) posits that "No one else matters". He refers to the Stickiness Factor as the content and quality of the message and asserted that the structure and format of the message can have profound effects on whether the idea spreads and is adopted or not. An example illustrated is the concept of educational pre-school television pioneered by Lesser and Cooney in the 1960s with Sesame Street using the then, new medium of television. Gladwell (2001) also alluded to the concept of context, which can shape "the course of social epidemics" (p. 259).

Much of the literature, however, seemed not to take cultural context into account in any great detail. In much of the literature reviewed, significant sample data were drawn from groups that were not dissected to determine if national or community contexts played significant roles. In exploring the application of Japanese management techniques, Leung Chee et al. (1994) found that transposing many of these techniques to Western corporations failed or only had limited success because they attempted to adopt only the technique and not the social context and philosophy that were integral to the success of the technology/techniques.

The diffusion of technology (Rogers, 1995; Moore, 1995; Norman, 1998; Soong, 2000) and the technology acceptance model (Davis, 1989; Kelleher \& O’Malley, 2006) are germane to this study as is indeed studies in SMS texting (Jordan \& Comrie, 2006) and various other related works are reviewed. Rogers (1995) suggested that not everyone adopted technological innovations at the same rate by identifying groups as innovators, early adopters, early majority, late majority, and laggards for illustration. Rogers' (1995) seminal theory of diffusion 
of innovation is regarded by Sooknanan (2006) as an innovation in itself owing to its overwhelming application in numerous studies.

Norman (1998) re-labeled the groups in keeping with technology market (customers) as innovators/technology enthusiasts, early adopters/visionaries, early majority/pragmatists, late majority conservatives and finally laggards/skeptics. He stressed that there was a virtual "chasm" (Moore, 1991, 1995) between the visionaries and the early majority. The model was used to discuss the reasons why marketing strategies and products need to change as the market matures since innovators drive the markets in the early stages while the majority drives it in the mature stage (see Figure 1).

On the other hand, Davis (1989 cited in Kelleher \& O'Malley, 2006) developed a Technology Acceptance Model that indicate perceived ease of use precedes perceived usefulness which has a direct effect on the individual's intentions and hence their usage behavior (see Figure 2).

Kelleher and O'Malley (2006) attempted to test whether this model would apply to public relations students at two Universities in the US and Korea and secondly, whether students' perceptions of online message boards would change after completing a class project that required the use of the technology. The results seemed to indicate that in order to cross the adoption barrier permanently the technology has to be applied in a constant environmental context so that adoption in one context or organization did not necessarily mean adoption in another context. However, should the context prove to be the same, then early adoption due to perceived ease of use and perceived usefulness might influence the rate of adoption or diffusion of the technology. All the participants were of roughly the same age with the same level of education and these demographics were eliminated from affecting that study.

In another study by Merrier and Dirks (1997), students' attitudes to oral, written and e-mail communication were investigated on a 10-point scale. E-mail got the highest score of 7.42, while written communication followed closely at 7.41 and oral trailed behind at 5.47. It is interesting to note that all communications scored above 5.0. Whether educational or age demographics can have an effect is unclear since it was largely a homogenous group from that perspective.

Jordan and Comrie (2006) conducted research into an attempt to introduce text-messaging technology to poll community opinion for use by the Auckland City Council of New Zealand with varying results. While the Council's Manager of Research and Consultation was pleased with the results, he said "Texting is biased towards young people (just as the usual process is biased against young people) and so residents didn't like the idea of young people being involved in the process. If we didn't consult with young people I think it would be

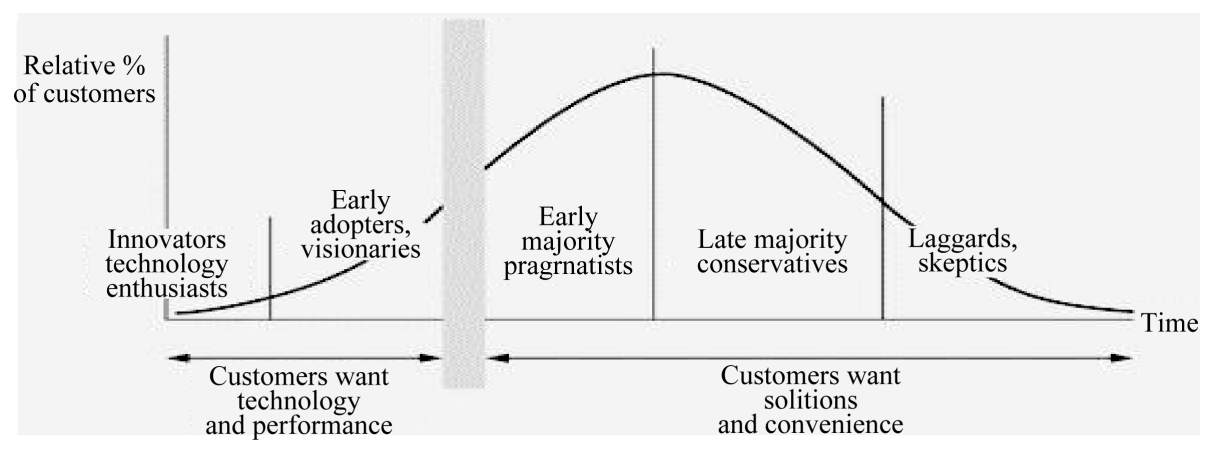

Figure 1. The chasm (Norman, 1998).

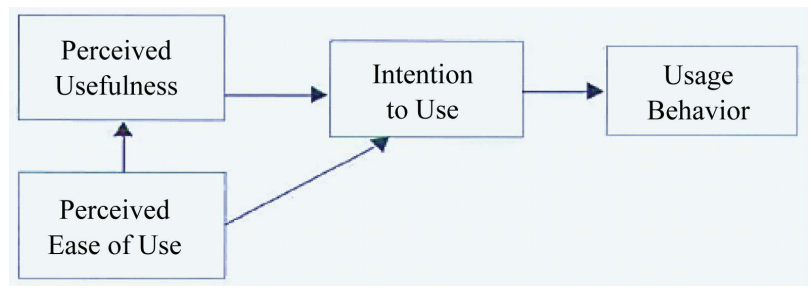

Figure 2. Technology acceptance model (Davis, 1989). 
illegal-under the Local Government Act 2002, part 6, section 82, 1 (b)”. It is interesting that the attitude towards text messaging was not the same as e-mail. This change perhaps signals that e-mail has moved into the realm of "mature" communication technology, while texting, message boards, and instant messaging are now the innovative technologies, at least in the developed world construct.

The implications for these results are significant since this would imply that even if technology was innovated by younger more technology savvy staff, it might still be a difficult road to wider adoption if the majority of staff and management perceived that the new technology might erode their ability to dominate the communication process.

On another note, Jackson (2007) posits that we are now entering into a "multicommunicating" environment where distinction between the different types of communication might become less meaningful as the various technologies mix with each other and with other more traditional communication methods. He argued that there are two levels of effects, the intended and the un-intended. An early example is given as e-mail, which was often implemented to distribute information effectively. However, second level effects such as rapid sharing of indiscreet information and supplanting various occasions of face-to-face communications (Sproull \& Kiesler, 1991; Jackson, 2007) arise un-intentionally.

\section{Setting and Context}

The Point Lisas energy-based companies located on the Point Lisas Industrial Estate are involved in three (3) main spheres of industry: 1) steel production, 2) fertilizer production (Ammonia/Urea), 3) methanol production. All three industries are fairly conservative with respect to new technologies. The overriding unstated philosophy is that in most cases, tried and tested technology is usually preferred. This is probably true with the exception of the "iron carbide" segment of the steel industry that is extremely innovative and experimental. This is evidenced by the relatively slow adoption of new technologies in comparison to other upstream energy counterparts, in the production, communications, and systems arena. There are some differences internal to Point Lisas among existing energy companies perhaps owing to cultural parenthood (respectively American/Canadian, Scandinavian and Trinidadian) as well as resources and global reach. A prominent one is well known for its accomplishments in e-auction technology in their procurement process and is an innovator locally for this technology. This company has conducted several seminars for government and has facilitated state enterprises in this technology. Two companies, on the other hand, are still on the "drawing board" with respect to this technology. The technology is not only a method for procurement but is indeed a virtual marketplace and a mechanism for communications between buyer and seller.

The energy company in this study has been innovative in its production technology resulting in expanded production thus demonstrating a capacity for innovation. However, in communication technology it seems to lag behind. Personal computers were not widespread as late as the late 1990s compared to its counterparts at the time that had been fully "computerized" by 1995. Further, not all managers are facilitated with mobile computing and even less professional staff. Very few professional staff have company mobile phones and only a few very select senior managers are facilitated with "Blackberry" technology. Personal computers are widely in use however and the company has both wide internet and intranet usage, including e-mail and internet availability. Mobile computers however are not widespread. The company comprises a mixture of staff from twenty-one years to fifty plus with the ratio of females to males less than ten percent (10\%).

In informal discussions across the company, management e-mail seems to be viewed by many as impersonal, and the company is largely driven by an oral communication culture. For example, in many meetings minutes are not taken. By comparison, informal discussions with staff in other multi-national companies (outside of Point Lisas) show marked differences in communications technology as well as communications culture.

Examples of this would be references made by interviewees at a major upstream energy company where communications culture was said to be a primarily "written culture" as opposed to oral. Interviewees indicated that e-mail is a standard of communication and that even oral communications is often followed up with a confirmation by e-mail. This is in marked contrast to the company in this study. An interviewee at a major light manufacturing company also indicated that not only do they have e-mail as a standard, but they have a technology adaptation of "instant messaging" which is a business communication technology used to regularly communicate internally and externally with sister companies. 


\section{Methodology}

The study focuses on the Procurement Department at an energy company in Trinidad. A self-administered survey of one hundred percent (100\%) of the staff was conducted (fourteen in total). The survey was balanced, containing seven (7) women and seven (7) men, with six (6) persons under the age of thirty five (35) and five (5) persons having a tertiary level education. Their communication preferences and tendencies were tested in diverse ways. Preferences for different types of communication included 1) verbal, one-on-one, 2) verbal, groups, 3) written memos, 4) e-mail, and 5) Short Messaging Service (SMS Text).

These communication preferences were tested in two ways: 1) a direct rated questions which were analyzed by taking average scores for different demographics, and 2) indicative questions such as use of computers and e-mail at home/personal life and at work. Stated preferences were also compared with the stated behavior at home to determine whether these were in line. Hence, some check on the subjectivity of the participant was therefore tested.

Indications of a tendency to be "early adopters" (Rogers, 1995) were investigated with questions that pertained to use of technology in personal life outside of the corporate environment. The rationale for this was that if an individual used technology outside of the structured corporate framework, he/she would more than likely be receptive to the technology if introduced at work.

Ownership of a home computer counted towards an overall score in this regard but additional points were scored if the individual also had personal e-mail addresses (separate from their work e-mail) and indicated that they used e-mail at home. If they owned a computer but answered negatively to either of the follow-up questions, they were scored lower since this indicated that perhaps the computer was used for purposes other than communication or that other family members were the actual users of the unit.

The sample is also significant in the local industry context as well. There are only four (4) producers of ammonia in Trinidad and Tobago with a collective procurement staffing of fewer than forty to fifty persons out of an estimated one thousand (1000) total staff (all departments). This study provides a one hundred percent (100\%) sample in terms of the subject department, and a sample of the representative Point Lisas ammonia producers total procurement staff of twenty to twenty-five percent $(20 \%-25 \%)$. While it is true that this sample will be from a homogenous corporate culture, having all the respondents from a single corporation ensures that corporate culture is eliminated from affecting the study and as such the results are more useful for the corporation itself. Notwithstanding, some generalizability is afforded especially in the wider energy sector.

\section{Data Analysis}

Use of the computer/e-mail were separated into two (2) age groups-under thirty five (35) (five persons) and over thirty five (35) (eight persons). Scores were allocated as follows:

I. Use of e-mail at work (1 point);

II. Use of e-mail at home (1 point);

III. Having a personal e-mail address (1 point);

IV. Having more than one personal e-mail (2 points);

V. Having a computer at home (1 point).

Following the compilation of the data, it was evident that the under thirty-fives scored consistently higher (three and above) (Figure 3).

(Note: the vertical scale indicates number of persons while the horizontal scale represents scores.)

With respect to whether each person had or did not have a personal e-mail, the results are even more conclusive (Figure 4). Clearly, while all persons under thirty-five (35) indicated positively, more than half of the over thirty-fives indicated they had no personal e-mail. It should be noted that the three respondents over thirty-five that had positive responses were all under fifty.

SMS (text) technology for mobile phones (and instant messaging technology) and the following scoring system were utilized:

i. Texting at work (1 point);

ii. Texting at home (1 point);

iii. Messaging at home (1 point);

iv. Would feel comfortable using instant messaging at work (1 point). 


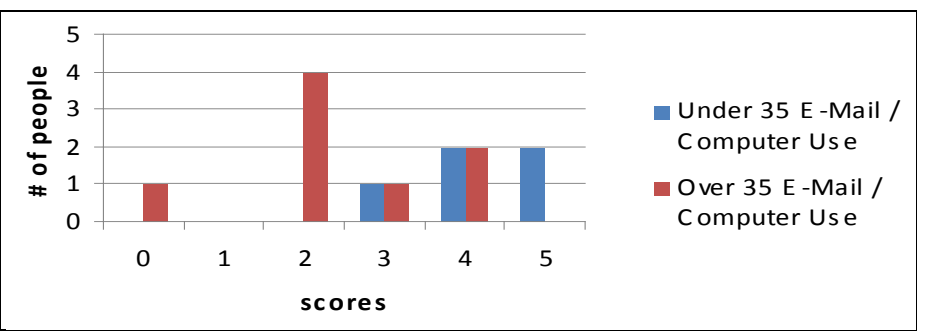

Figure 3. Age and e-mail/computer use.

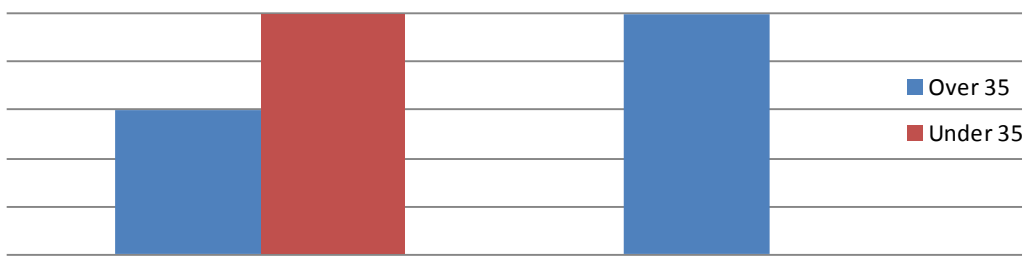

Figure 4. Age and personal e-mail.

The results were inconclusive with a wide spread for the under thirty-fives. However, the over thirty-fives had over half of the respondents (five) scoring two and under (Figure 5).

Respondents were also asked to give scores for the following preferences to various communication methods:

(i) Written memos;

(ii) One-on-one meetings;

(iii) Group meetings;

(iv) E-mail;

(v) SMS texts.

A score of five indicated a high preference and a score of one indicated a low preference. The scores are tabulated in Figure 6 (by age) and Figure 7 (by education) below. The averages were taken in each group so that they could be compared.

In all areas, the under thirty-fives scored higher and the tertiary respondents scored higher in all areas except written memos lending suooprt to the position taken by Merriera and Dirks (1997). This tends to indicate that generally the under thirty-fives seemed to value communications in general more than the over thirty-fives. This can be attributed to greater tendency to socialize or the need to communicate.

Finally, a comparison (Figure 8) was done to look at secondary or lower versus tertiary level respondents with regards to having personal e-mail addresses. All the tertiary level respondents were positive while more than half of the non-tertiary level respondents were negative.

\section{Discussion}

Based on the demographics of age and education, it is evident that younger respondents valued communications more and had higher tendencies to use communication technology in their personal lives. The results were similar for tertiary level respondents having similar characteristics of the younger age demographics. This suggests that at the Procurement Department of this energy company, younger staff with tertiary education would tend to be the "innovators" or "early adopters" (Rogers, 1995) and would help drive the diffusion process. These staffers would be the ones to assist in moving the group across the "chasm" (Moore, 1995). These characteristics are endorsed by Soong (2000) who asserts that the early adopters came from the higher socio-economic levels (ten percent at the highest level) and more importantly by higher levels of education (thirty percent at the highest level).

This study, therefore, supports the conventional literature. As a result, in order to boost the level of innovation and speed, the diffusion of communicative technology, increasing the numbers of younger, higher educated staff would be a valid strategy. In fact, these individuals would become the "change agents" who would facilitate the 


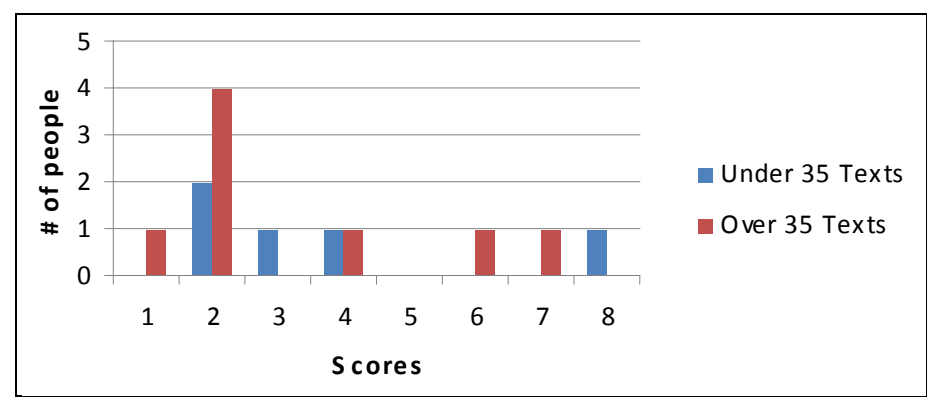

Figure 5. Age and use of SMS texting.

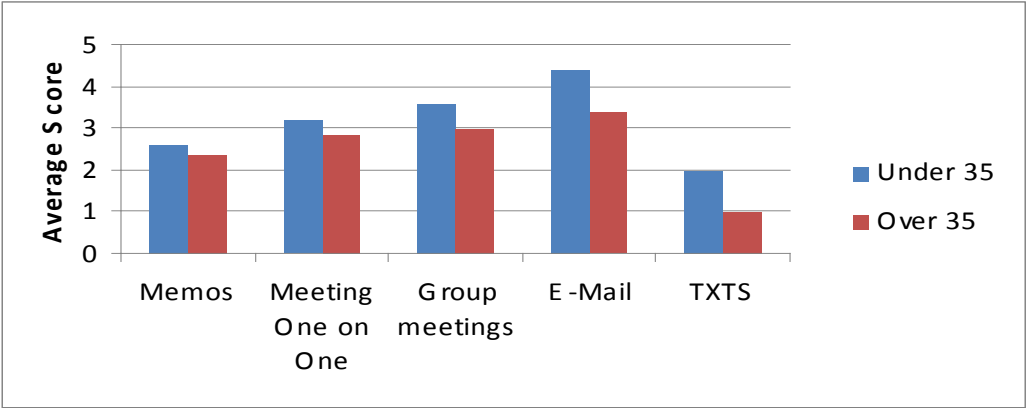

Figure 6. Preferences by age.

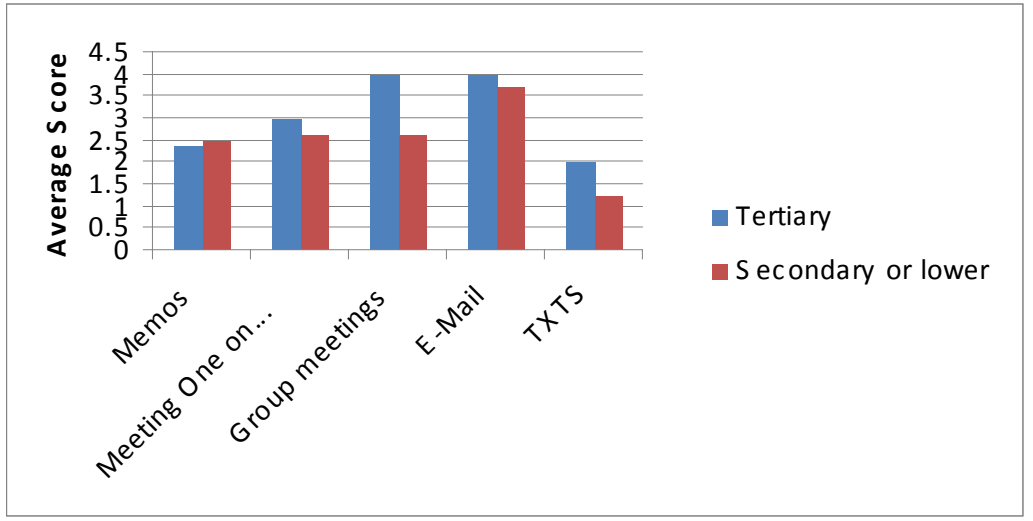

Figure 7. Preferences by education.

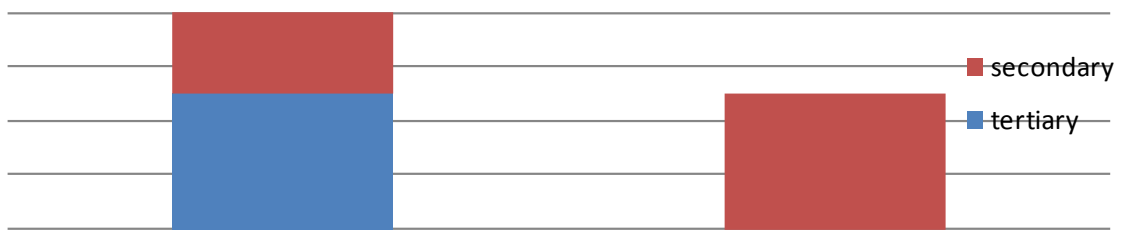

Figure 8. Education and personal e-mail.

change process. However this has to be tempered by the evidence offered by Jordan and Comrie (2006) who suggest that for this strategy to be fully effective it would have to be championed by a significant portion of the management group as well as prominent members of the older age group. Closely related would be the application of the tipping point theory (Gladwell, 2001), where it would be advisable to seek out the organizational 
connectors, mavens and salesmen. Relatedly, it might be worthwhile to group education level and age together as one demographic without testing correlation since only two out of the eight over thirty-fives had tertiary level education and in those cases the respondents were under fifty.

In a largely conservative social construct, the factors that can affect the rate of innovation and diffusion of communication technologies such as competitive advantage depend on a number of factors. For example, age and educational level are likely to influence the innovation process. However, the rate of adoption and diffusion cannot be totally overcome by infusing these demographics alone. More than likely, it will also depend on finding and selecting champions who are representative of the social status quo to attain the state of "critical mass" necessary for successful diffusion.

Age and education levels are likely to influence the chances of innovation and rapid diffusion of communications technology in the energy environment in Trinidad and Tobago and possibly in other like environments. However, this will probably only be successful if the technologies are sufficiently championed by those who hold greater social status. Some keys to success would be ensuring that the technology is perceived as easy to use, as having usefulness and are managed so as not to shift the balance of influence away from older more established staff. The organization's demographics in terms of age and educational level is, therefore, supremely important in determining any strategy to innovate or implement any new technologies, communication or otherwise.

The findings of the study support all of the models reviewed in the literature review. In fact, the merging of techniques (Jackson, 2007) is seen in even the preferences for various communication techniques/technology. The Technology Diffusion model (Rogers, 1995) is supported by the emergence of clear self-directed users of the technology in personal life, while the Technology Acceptance Model (Kelleher, 2006) is clearly supported by the intent and continued use of technologies in personal lives that spill over into the office as opposed to only occurring within the work structure. The work of Jordan and Comrie (2006) is particularly relevant as it provides a context and theory as to why older staffers might be laggards or skeptics (Rogers, 1995). While there might be a tendency to attribute success to education and exposure alone, it might be safer to think of the problem as more related to status quo, power and influence.

The research questions (RQs) for the study were as follows:

RQ 1: Do age and education demographics affect technology acceptance and adoption within a particular corporate environment?

RQ 2: Can infusing a group with younger more educated staff assist with speeding the diffusion process?

Clearly age and education level can play a role in the diffusion process in the subject organization. These demographics can influence the innovation process as well as the early adoption process. If the young and tertiary educated demographics are dominant it may also have a faster diffusion process. However, where it is not dominant then other issues such as second level effects (un-intentional), status quo concerns etc. may become greater concerns and require other measures to avert the barriers that arise. These may include the mode of presentation and identifying the individuals in the organization that hold the social power to bring about change.

The other research question in the study was:

RQ 3: Does usage of the technology at home correlate with more positive attitudes towards such technologies in the work place?

The study does tend to show that usage of technology in personal life seems to always translate into positive usage or attitude to the technology at work. The younger staff tended to utilize the technologies in their personal lives more than the older staff.

\section{Conclusion}

While this study is particularly relevant to the Procurement Department of an energy company in Trinidad and Tobago, it would need to be expanded to a sample of the wider organization or other companies to yield more generalizable data. In expanding to a wider cross organizational or national level, the impact of cultural norms in countries as well as communities and organizations themselves may influence outcomes and as such it may be useful to keep like-groups separated for individual analysis and then amalgamate them for further, wider analysis.

Expansion of the study outside of the organization would be of academic interest, as it would produce data that could relate to a national cultural context rather than just an organizational context. This could inform re- 
cruitment indicators as well as inform tertiary and secondary institutions of the relevance of the infusion of such technologies into the school curriculum. On a national scale this could influence the rate of diffusion on a national level, giving organizations that operate in the national context a competitive advantage.

The study, therefore, in the context of a case study provides useful strategic information for a particular corporate department. These findings can be compared to other similar type and sized departments. In addition, the study can be extended to compare the effect of different corporate cultures and leadership styles. Further, it can be expanded on a national level as a pathway to competitive advantage in a growing global energy environment.

\section{References}

DeFleur, M. H., Kearney, P., Plax, T. G., \& DeFleur M. L. (2005). Fundamentals of Human Communication (3rd ed.). New York: Mc Graw Hill.

Gladwell, M. (2001). The Tipping Point. New York: Bay Back Books.

Jackson, M. (2007). Should Emerging Technologies Change Business Communication Scholarship? University of Colorado Boulder. Journal of Business Communication, 44, 311-324.

Jordan, A., \& Comrie, M. (2006). Texting and Other Forms of Communication in Local Government Consultation. Massey University, The Communication Journal of New Zealand, 6, 191-224.

Kelleher, T., \& O’Malley, M. (2006). Applying the Technology Acceptance Model to Assess Outcomes in a Globally Linked Strategic Communication Project. Journalism and Mass Communication Educator, 402-414.

Leung Chee, J., Loquan, M., \& Lau, S. (1994). Japanese Management Techniques and Philosophy-JIT Implementation in Trinidad. 8th Annual Conference-APETT (Association of Professional Engineers).

Merrier, P. A., \& Dirks, R. (1997). Student Attitudes toward Written, Oral and E-Mail Communication. Business Communication Quarterly, 60, 208-218.

Moore, G. A. (1991). Crossing the Chasm: Marketing and Selling High-Tech Goods to Mainstream Customers. New York: Harper Business.

Norman, D. A. (1998, 2004). The Lifecycle of a Technology. Nielsen Norman Group Report. http://www.jnd.org/dn.mss

Rogers, E. M. (1995). Diffusion of Innovations. New York: The Free Press.

Sooknanan, P. (2006). Attitudes and Perceptual Factors in the Adoption of Computers in a School System: A Case Study of Trinidad and Tobago. Journal of Creative Communications, 1, 235-251.

Soong, R. (2000). Early Adopters of Technological Innovations. Zona Latina. http://www.zonalatina.com/zldata99.htm

Sproull, L., \& Kiesler, S. (1991). Connections: New Ways of Working in the Networked Organization. Cambridge, MA: MIT Press. 
Scientific Research Publishing (SCIRP) is one of the largest Open Access journal publishers. It is currently publishing more than 200 open access, online, peer-reviewed journals covering a wide range of academic disciplines. SCIRP serves the worldwide academic communities and contributes to the progress and application of science with its publication.

Other selected journals from SCIRP are listed as below. Submit your manuscript to us via either submit@scirp.org or Online Submission Portal.
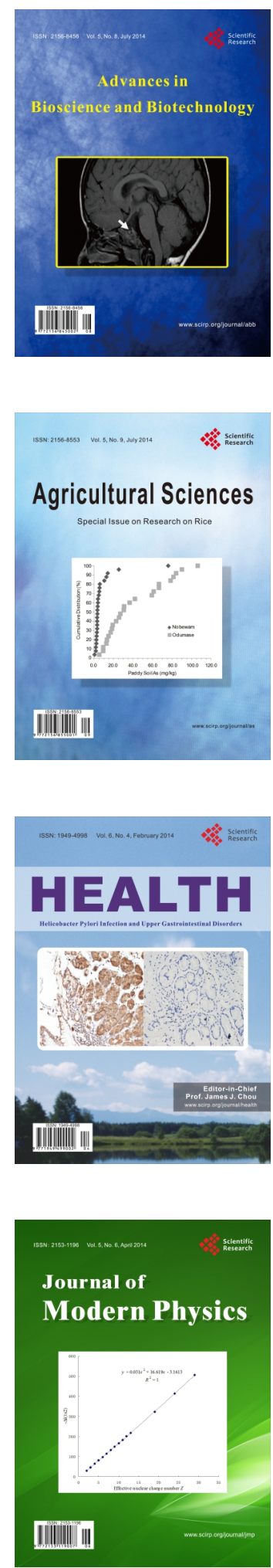
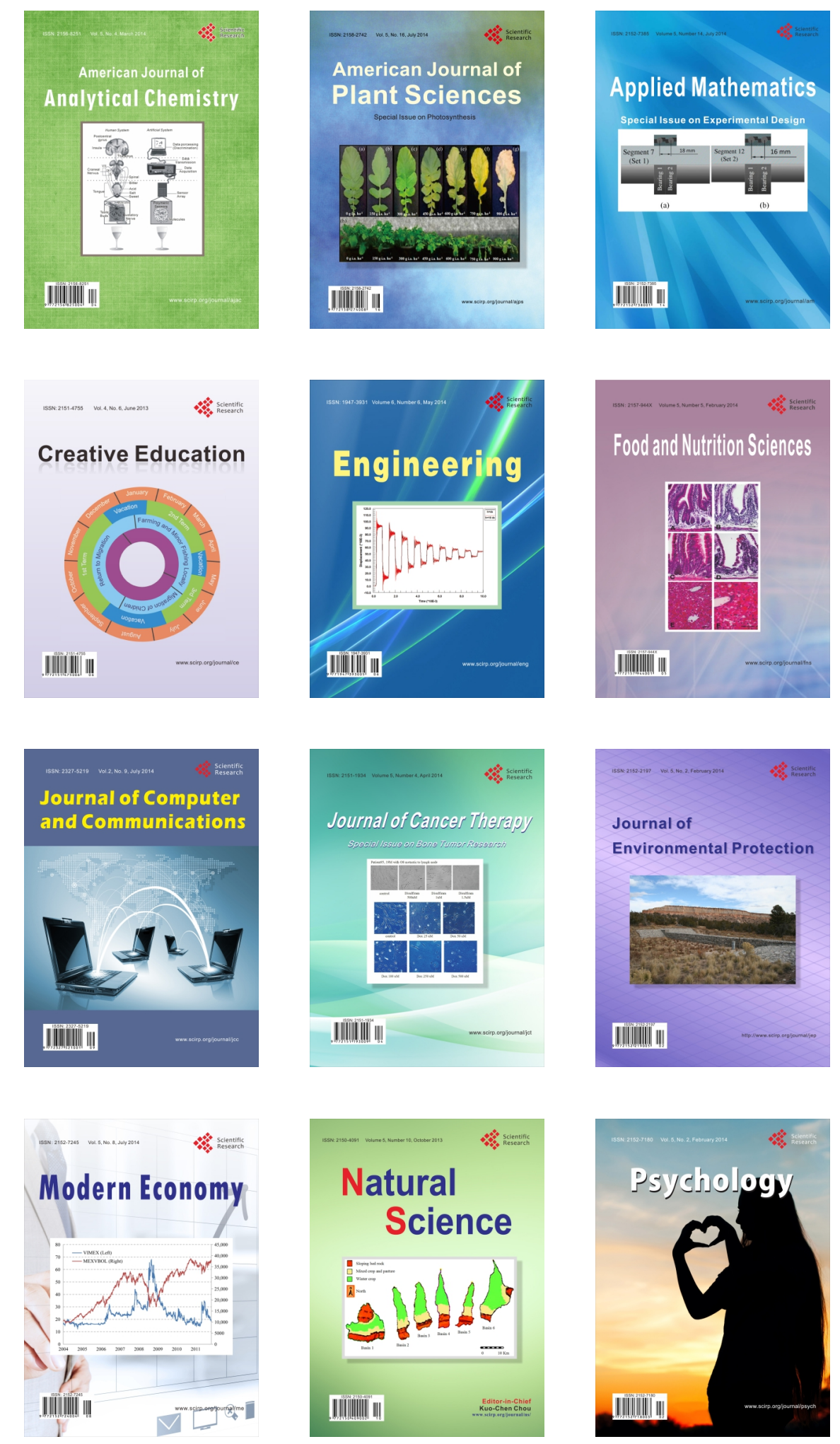